\title{
CRECIMIENTO DE Fraxinus uhdei INOCULADO CON DOS CEPAS ECTOMICORRÍZICAS EN DOS SUSTRATOS, UNO CONTAMINADO CON MERCURIO
}

\author{
Growth of Fraxinus uhdei inoculated with two ectomicorrhyzic strains in two substrates, one polluted with mercury
}

\author{
Ivett PÉREZ BALTAZAR ${ }^{1}$ Ana Laura BÁEZ-PÉREZ², Verónica OSUNA-VALLEJO², \\ Cynthia ARMENDÁRIZ-ARNEZ ${ }^{1}$ y Roberto LINDIG-CISNEROS ${ }^{2 *}$
}

\author{
${ }^{1}$ Laboratorio de Contaminación y Salud Ambiental, Escuela Nacional de Estudios Superiores, ENES-Morelia, \\ UNAM, Antigua Carretera a Pátzcuaro 8701, Ex-hacienda de San José de la Huerta, 58190 Morelia, Michoa- \\ cán, México \\ ${ }^{2}$ Laboratorio de Restauración Ambiental, Instituto de Investigaciones en Ecosistemas y Sustentabilidad, UNAM. \\ Antigua Carretera a Pátzcuaro 8701, Ex-hacienda de San José de la Huerta, 58190 Morelia, Michoacán, México \\ *Autor para correspondencia: rlindig@iies.unam.mx
}

(Recibido: febrero 2019; aceptado: septiembre 2019)

Palabras clave: minería, asociación simbiótica, contaminación, metales pesados

\begin{abstract}
RESUMEN
La minería es una actividad industrial importante en muchos países, a pesar de lo cual los daños que causa son cada día más graves por su impacto ecológico. Lo anterior se debe, entre otras cosas, a los efectos de los residuos de metales pesados originados por los diversos métodos de extracción de minerales. Para reducir el impacto ambiental de esta actividad es importante estudiar relaciones simbióticas entre especies que permitan favorecer la supervivencia y el desarrollo de plantas en sitios degradados por la minería, y, de esta manera, mejorar su estructura al incorporar materia orgánica y reiniciar ciclos biogeoquímicos. Por lo tanto, se llevó a cabo un ensayo en mesocosmos para documentar la respuesta de Fraxinus uhdei (Wenz.) Lingelsh (fresno) inoculado con hongos ectomicorrízicos en dos sustratos diferentes: uno proveniente de un jal del municipio de Tlalpujahua de Rayón, Michoacán, sitio donde se desarrolló actividad minera; y otro orgánico. El experimento consistió en someter a individuos de F. uhdei a inoculación con Laccaria laccata y Lactarius deliciosus con dos dosis: $2.5 \times 10^{5}$ y $1 \times 10^{6}$ de esporas por planta). Los hongos ectomicorrízicos favorecieron el desarrollo y supervivencia de las plantas en ambos sustratos. El desempeño de las plantas fue mayor en el sustrato orgánico, ya que la altura, cobertura y peso fresco de las plantas inoculadas con Laccaria laccata fueron mayores $\left(66 \mathrm{~cm}, 1186 \mathrm{~cm}^{2}\right.$ y $92 \mathrm{~g}$, respectivamente) respecto a los otros tratamientos. La concentración de mercurio fue menor para las plantas inoculadas con Lactarius deliciosus $\left(\mathrm{F}_{(1,28)}=12.22 ; \mathrm{p}=0.002\right)$ respecto a las inoculadas con Laccaria laccata. Este estudio indica que la inoculación favorece el desarrollo de plantas de fresno tanto en un sustrato orgánico como en un sustrato derivado de la actividad minera; asimismo, exhibe el potencial de la especie arbórea para la recuperación de sustratos mineros.
\end{abstract}

Key words: mining, symbiotic association, pollution, heavy metals 


\begin{abstract}
Mining is one of the most important industrial activities in many countries; however, environmental damages associated with this activity are severely detrimental. These effects are a consequence, among other things, of heavy metals originated by mineral extraction methods. To reduce the environmental impact of this activity, it is important to develop remediation technologies, including the symbiotic relationships between species that allow high survival and proper development of plants. Therefore, a mesocosm assay was carried out to document the response of Fraxinus uhdei (Wenz.) Lingelsh (ash) inoculated with ectomycorrhizal fungi on two different substrates: a mine-derived substrate from the municipality of Tlalpujahua de Rayón, Michoacán, and an organic one. The study consisted in the inoculation of $F$. uhdei plants with two fungi strains, Laccaria laccata and Lactarius deliciosus, on two doses: $2.5 \times 10^{5}$ and $1 \times 10^{6}$ spores per plant. Ectomycorrhizal fungi favored the development and survival in both substrates; however, the performance of the plants was higher with the organic substrate. Height, cover, and fresh weight of plants inoculated with Laccaria laccata were the highest $\left(66 \mathrm{~cm}, 1186 \mathrm{~cm}^{2}\right.$, and $92 \mathrm{~g}$, respectively). Mercury was lower in plants inoculated with Lactarius deliciosus $\left(\mathrm{F}_{(1,28)}=12.22 ; \mathrm{P}=0.002\right)$ with respect to those inoculated with Laccaria laccata. Our results indicate that inoculation has a positive effect on ash growth both in an organic rich substrate and a substrate produced by mining activities, also the potential use of the tree species for remediation of mining dumps.
\end{abstract}

\section{INTRODUCCIÓN}

Una de las actividades económicas más importantes en México y otros países es la minería. Esta actividad ha sido fundamental a lo largo de la historia, ya que ha determinado la dinámica económica y social de los sitios en que ha prosperado (Muñoz 1986). Desde la época colonial hasta finales del siglo XIX, la producción minera en México tuvo un desarrollo muy importante que favoreció las actividades económicas y, por ende, sociales del país. Además, provocó diversos impactos ambientales, paisajísticos y de salud pública, cuyos efectos continúan manifestándose hasta nuestros días (Uribe 2002).

A partir de 2011, la producción de plata y oro recobró su auge en México, llegando a representar el $56 \%$ de la producción metalúrgica nacional con una alta inversión extranjera. Además, para 2017 contribuyó con el $4 \%$ del PIB nacional (Góngora 2013), lo cual muestra la importancia de entender los impactos ambientales que se relacionan con esta actividad y la forma de mitigarlos.

Específicamente, en el municipio de Tlalpujahua de Rayón, localizado entre las coordenadas $19^{\circ} 41^{\prime}-19^{\circ}$ $53^{\prime}$ de latitud norte y $100^{\circ} 08^{\prime}-100^{\circ} 18^{\prime}$ de longitud oeste en el estado de Michoacán, se llevó a cabo una fuerte explotación minera durante el período colonial tardío y hasta inicios del siglo XX (Corona et al. 2010). Como consecuencia de esta actividad cambiaron tanto el paisaje, debido a la deforestación y erosión, como la disponibilidad de elementos químicos en el suelo a causa de los residuos mineros, lixiviación y formación de nuevos minerales. Por ello el municipio presenta afectaciones ambientales, entre ellas la presencia de depósitos de desechos mineros conocidos como jales. Éstos tienen impactos en la población y la dinámica del ecosistema, principalmente por la contaminación con metales pesados que los acompañan, además de su propia estructura, la cual dificulta el desarrollo de vegetación (Corona et al. 2017).

Durante aproximadamente 350 años se utilizó para la extracción de oro y plata el método de amalgamación con mercurio o método de patio, que consiste en mezclar en un patio mercurio metálico $(\mathrm{Hg})$, sal, cobre, hierro, agua y los minerales triturados para dejarlos reposar días o semanas. Posteriormente se separa la amalgama de oro, plata y mercurio mediante un proceso de lavado, evaporación y calentamiento que volatiliza y recupera parcialmente este último metal. Así fue como el mercurio se incorporó a los suelos del municipio. Además, las arenas y limos obtenidos de la trituración quedaron compactados en los sitios de producción, creando condiciones no aptas para el desarrollo de la vegetación (Lacerda 1997, Uribe 2002, Corona et al. 2010).

Uno de los retos para la rehabilitación de minas es la selección de especies para la revegetación (Singh et al. 2004), proceso fundamental para mejorar las condiciones del sustrato y facilitar el establecimiento de especies nativas a mediano y largo plazo (Tucker y Ungar 2002). En algunas ocasiones es necesario usar enmiendas o aditivos para el sustrato de tal 
manera que se facilite el establecimiento de las plantas (Brown et al. 2007). Otros estudios se han centrado en la identificación de barreras para el establecimiento de la vegetación; de ellos se desprende la dificultad de recrear las condiciones idóneas para el sitio a restaurar. Esto es consecuencia de que los terrenos degradados por actividad minera son muy susceptibles a presentar valores de $\mathrm{pH}$ alejados de la neutralidad y bajas concentraciones de elementos como nitrógeno $(\mathrm{N})$ y fósforo $(\mathrm{P})$, además de escasez de materia orgánica (Jim 2001).

Un aspecto menos estudiado es el efecto de las relaciones simbióticas como la micorrización para mejorar el crecimiento y desarrollo de las plantas destinadas a la rehabilitación de sustratos mineros. En particular, la micorrización puede facilitar el desarrollo de plantas en sustratos con baja disponibilidad de nutrientes, además de facilitar la absorción de agua mediante la formación de agregados del suelo, creando un suelo poroso y permeable (Royo et al. 1998, Crane et al. 2012, Pereira et al. 2014).

Por ello en este estudio se analizó la relación simbiótica entre los hongos ectomicorrízicos (HEM) Lactarius deliciosus y Laccaria laccata con Fraxinus uhdei, especie arbórea nativa de México (fresno). Esta especie tiene importancia económica y es tolerante a sustratos pobres (Conabio 2017), por lo que probar su utilidad para fitorremediar suelos contaminados es una opción atractiva.

El objetivo central de esta investigación fue analizar la respuesta de Fraxinus uhdei a la presencia de mercurio $(\mathrm{Hg})$ en dos sustratos contrastantes y con dos especies diferentes de HEM, a través de un experimento en mesocosmos. Se planteó la hipótesis de que la inoculación del fresno con hongos ectomicorrízicos mejora el desarrollo, supervivencia y resistencia de esta especie cuando se desarrolla en sustratos contaminados con Hg. Esta hipótesis está sustentada en que se ha encontrado que diversas especies de ectomicorrizas son capaces de almacenar metales pesados depositados en suelos contaminados y que, al hacerlo, reducen el daño que estos contaminantes causan a las plantas (Brown y Wilkins 1985, Dermibas 2001).

\section{MATERIALES Y MÉTODOS}

\section{Material vegetal}

Se propagaron 300 plantas de fresno en condiciones de casa de sombra (20\% de reducción de la intensidad lumínica), sembradas en contenedores de plástico $\left(240 \mathrm{~cm}^{3}\right)$, utilizando como sustrato agrolitaturba 2:1 (v:v), esterilizada en autoclave por $30 \mathrm{~min}$ a $121^{\circ} \mathrm{C}$ y 15 libras por pulgada cuadrada (PSI, por sus siglas en inglés). A las siete semanas de crecimiento, se seleccionaron al azar 200 plantas y fueron inoculadas a una profundidad de $3 \mathrm{~cm}$ cerca de la raíz con dos especies de HEM, Laccaria laccata y Lactarius deliciosus, provenientes de zonas boscosas de la localidad de Yoricostio, Tacámbaro, Michoacán.

\section{Preparación de inóculo e inoculación}

Los inoculantes se prepararon como se describe a continuación. Después de la identificación de las especies micorrízicas del material recolectado, los cuerpos fructíferos fueron deshidratados y molidos (el tamaño de las partículas se homogeneizó con un tamiz de $0.50 \mathrm{~mm}$ ). Se determinó la concentración de esporas viables en el inoculante con una cámara de Neubauer, previamente tiñéndolas con sales de tetrazolio (MTT). Este reactivo permite evaluar la actividad metabólica celular, ya que un color azul indica esporas metabólicamente activas (Báez-Pérez et al. 2015). El inóculo se diluyó en turba micronizada para su posterior aplicación, de tal forma que por cada gramo de turba micronizada hubiera al menos $1 \times 10^{6}$ esporas del hongo micorrízico. Los tratamientos de inoculación fueron de $2.5 \times 10^{5}$ y $1 \times 10^{6}$ esporas/ planta. Estudios previos determinaron que estas dosis son apropiadas para lograr la micorrización de las plantas de fresno (Báez-Pérez et al. 2017). La micorrización se evaluó con la técnica de azul de tripano a una concentración de $0.05 \%$ por $48 \mathrm{~h}$, descrita por Báez-Pérez et al. (2015).

\section{Establecimiento del experimento}

Se realizó una selección completamente al azar de los árboles propagados e inoculados de fresno para ser distribuidos entre los dos sustratos, el del jal extraído de Tlalpujahua de Rayón, localizado al noroeste del estado de Michoacán, y el orgánico que se elaboró mezclando materia orgánica (corteza de pino molida) con suelo de una parcela agrícola obtenido de la misma localidad. El experimento consistió en un diseño de dos factores. El primero, la inoculación con tres niveles (Laccaria laccata, Lactarius deliciosus y testigo sin inocular), y el segundo, el sustrato con dos niveles, en un diseño ortogonal con 15 plantas por cada uno de los seis tratamientos resultantes de la combinación de factores.

El experimento se llevó a cabo en el Laboratorio de Restauración Ambiental del Instituto de Investigación en Ecosistemas (IIES), en un sistema de mesocosmos, es decir, en pequeño, en el cual se intentó simular las funciones de un ecosistema natural (González 2012). 


\section{Monitoreo del experimento y análisis de mercurio}

Las plantas fueron monitoreadas mensualmente en alturas y trimestralmente en cobertura y diámetro a la altura de la base (DAB). Se registraron dos diámetros de la copa de las plantas y se calculó la cobertura por medio de la siguiente fórmula (Popma et al. 1988):

$$
0.25 \times \mathrm{D} 1 \times \mathrm{D} 2 \times \pi
$$

Asimismo, se calculó el error estándar $(\sigma / \sqrt{n})$ para cada promedio de las variables de crecimiento.

La determinación de contenido de $\mathrm{Hg}$ en los tejidos vegetales se hizo con espectrometría de emisión óptica (ICP-OES). Las muestras se prepararon con el método de digestión ácida. Para ello, las hojas de fresno de cada tratamiento se sometieron a un proceso de secado en horno por $48 \mathrm{~h}$ a una temperatura constante de $65^{\circ} \mathrm{C}$. Las muestras se pulverizaron en un mortero de cerámica para homogeneizarlas. Luego se utilizaron $0.4 \mathrm{~g}$ de muestra para la digestión con $5 \mathrm{~mL}$ de ácido nítrico $\left(\mathrm{HNO}_{3}\right)$ ultrapuro $(60 \%)$ y se calentaron en horno de microondas por $30 \mathrm{~min}$. Al terminó de dicho procedimiento la muestra se aforó a $25 \mathrm{~mL}$ y por último se colocó en tubos de lectura. Los procedimientos de control de calidad (QC, por sus siglas en inglés) y de confiabilidad (QA) se llevaron a cabo usando muestras que incluyeron calibración externa y se verificaron cada 20 mediciones con alícuotas del blanco. Para verificar la precisión, alícuotas de un material de referencia certificado (Crescent Chemical, Single-Element Standard en una matriz de $\mathrm{HNO}_{3}$ al $2 \%$ ) fueron sometidas al mismo procedimiento que el resto de las muestras. El límite de detección obtenido (LOD, por sus siglas en inglés) se calculó con la suma de las mediciones de los blancos y tres veces el valor de la desviación estándar. Para las muestras blanco el valor fue de $0.03 \mathrm{ppm}$ y para las muestras de digestión de 0.90 ppm. Los valores de recuperación se determinaron analizando muestras del blanco con concentraciones conocidas de $\mathrm{Hg}(0.05,0.1,0.5$ y $1.0 \mathrm{ppm})$. Para cada concentración se realizaron 10 repeticiones. La media de los valores de estas repeticiones se usó para calcular la recuperación de acuerdo con la siguiente ecuación (Eurachem Working Group 1998):

Recuperación $(\%)=([\mathrm{c} 1-\mathrm{c} 2] / \mathrm{c} 3) \times 100$

donde cl es la concentración de $\mathrm{Hg}$ en la muestra de concentración conocida, c2 es la concentración de $\mathrm{Hg}$ en la muestra de la matriz del blanco y c3 es la concentración adicionada de $\mathrm{Hg}$.
Los valores de recuperación de $\mathrm{Hg}$ para las concentraciones de $0.05,0.1,0.5$ y $1.0 \mathrm{ppm}$ variaron de 90 a $99 \%$, que es un rango aceptable para este elemento (Yogesh et al. 2015).

Los detalles de las condiciones de operación instrumentales se presentan en el cuadro I. Las curvas de calibración se obtuvieron con estándares de calibración preparados en el mismo medio ácido que las muestras. A pesar de la complejidad de la matriz analizada, no fue necesaria una calibración de adición estándar. La calibración se verificó cada 20 mediciones y la precisión de la técnica analítica se determinó sometiendo alícuotas de material de referencia certificado (Crescent Chemical, Single Element Standard en una matriz de $\mathrm{HNO}_{3}$ al 2 \%) al mismo tratamiento que las muestras analizadas.

CUADRO I. PARÁMETROS DE OPERACIÓN DEL EQUIPO DE ESPECTROMETRÍA DE EMISIÓN ÓPTICA (ICP-OES)

\begin{tabular}{lc}
\hline Parámetros del método & \\
\hline Potencia del generador de Radio frecuencia $(\mathrm{kW})$ & 1 \\
Flujo de plasma (L/min) & 12.8 \\
Flujo auxiliar (L/min) & 0 \\
Presión del nebulizador $(\mathrm{kPa})$ & 3.4 \\
Tiempo de toma de muestras $(\mathrm{s})$ & 30 \\
Tiempo de enjuague $(\mathrm{s})$ & 40 \\
Velocidad de la bomba (rpm) & 15 \\
Tiempo de replicado (s) & 15 \\
Tiempo de estabilizado (s) & 20 \\
Réplicas & 3 \\
Longitud de onda Hg (nm) & 253.652 \\
\hline
\end{tabular}

El grado de estrés de las plantas se estimó por medio del contenido de clorofila. Una hoja de cada individuo se tomó de la rama dominante, en la tercera ramificación a partir del ápice, para asegurar que fueran hojas maduras. Las hojas se dispusieron en tubos de ensaye añadiéndoles $10 \mathrm{~mL}$ de alcohol absoluto grado analítico y se colocaron tapadas en un recipiente cubierto con aluminio para no someterlas a la luz. Las muestras se dejaron reposar durante dos días a temperatura ambiente, para posteriormente aforarlas a $10 \mathrm{~mL}$. Las muestras con la clorofila extraída se leyeron en un espectrofotómetro Genesys 20 (Thermo Scientific) a 664 y $649 \mathrm{~nm}$. Las hojas se dejaron reposar para lograr la evaporación del alcohol y se pesaron en tres ocasiones o hasta lograr un peso constante. Para obtener los valores de clorofila a, b y su proporción se aplicaron las siguientes formulas desarrolladas por Lichtenthaler (1987): 
$\mathrm{Ca}=($ valor obtenido en el nivel de absorbancia de $649 \mathrm{~nm}) \times 22.24 \times$ (peso de la hoja en $\mathrm{g}$ ) $\times 1000$

$\mathrm{Cb}=($ valor obtenido en el nivel de absorbancia de $664 \mathrm{~nm}) \times 5.24 \times$ (peso de la hoja en $\mathrm{g}) \times 1000$

Proporción $\mathrm{a} / \mathrm{b}=$ valor obtenido en clorofila a/clorofila $b$

Las plantas se cosecharon a los 11 meses de iniciado el ensayo para determinación del peso fresco y seco (secado en estufa a $60{ }^{\circ} \mathrm{C}$ por $48 \mathrm{~h}$ ) de la parte aérea.

El sistema radical se examinó al microscopio debido a que las ectomicorrizas hacen simbiosis con las raíces de las plantas y éstas experimentan una modificación morfológica. Las raíces seleccionadas al azar se colocaron en una caja de Petri y cuales se tiñeron con azul de tripano al $0.05 \%$ por $48 \mathrm{~h}$. Antes de ser observadas al microscopio se les retiró el exceso de colorante con agua destilada, quedando teñidos tanto el manto como el micelio externo de los hongos asociados a los segmentos finos de las raíces de fresno (Báez-Pérez et al. 2015).

\section{Análisis estadístico de los datos}

Se realizaron análisis estadísticos a fin de determinar las diferencias entre tratamientos por medio de análisis de varianza factorial. Si las interacciones o el efecto de algún factor no fueron significativos se procedió a la simplificación del modelo siguiendo el procedimiento sugerido por Crawley (2007). Las comparaciones múltiples de medias se llevaron a cabo con la prueba de Tukey $(\mathrm{p}<0.05)$.

El análisis estadístico para clorofila y contenido de $\mathrm{Hg}$ en los tejidos se llevó a cabo con un análisis de varianza (ANDEVA) de dos factores. Los análisis se llevaron a cabo utilizando los paquetes estadísticos S-PLUS 2000, JMP 6.0.0 y R 3.4.3.

\section{RESULTADOS}

La inoculación de las plantas de fresno con Laccaria laccata o Lactarius deliciosus fue efectiva, ya que se observaron estructuras propias de los HEM como micelio externo septado y la red de Hartig después de la tinción con azul de tripano (Fig. 1).

En las figuras 2 y $\mathbf{3}$ se presentan las respuestas a lo largo del tiempo en altura, cobertura y $\mathrm{DAB}$, de

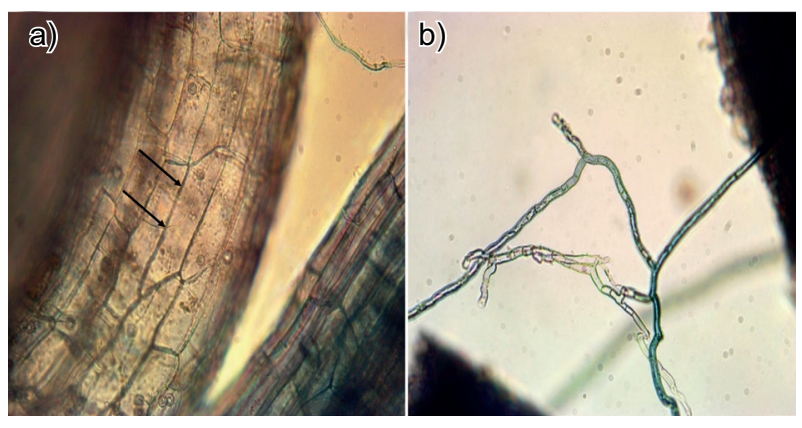

Fig. 1. Imágenes de las estructuras de hongos micorrízicos en asociación con Fraxinus uhdei, en (a) células corticales cubiertas por la red de Hartig y (b) micelio externo de la raíz de Fraxinus uhdei

las plantas de fresno inoculadas con Laccaria laccata y Lactarius deliciosus, respectivamente. En el cuadro II se muestran los valores finales para cada tipo de inoculación. El efecto del sustrato fue evidente. La altura de las plantas inoculadas con Laccaria laccata fue de $50 \mathrm{~cm} \pm 5.7$, la mayor en el sustrato orgánico con dosis alta de inóculo. Las plantas mostraron el mayor $\mathrm{DAB}, 11 \mathrm{~cm} \pm 0.8$, con la dosis baja. Para las plantas inoculadas con Lactarius deliciosus las variables de crecimiento fueron mayores en el sustrato orgánico y con la dosis baja de inoculación, para todas las variables de crecimiento: altura $(69 \mathrm{~cm} \pm 4)$, cobertura $\left(1094 \mathrm{~cm}^{2}\right.$ $\pm 97.3), \mathrm{DAB}(18 \mathrm{~cm} \pm 1.2)$, peso fresco $(83 \mathrm{~cm} \pm 9.6)$ y peso seco $(35 \mathrm{~cm} \pm 4.5)$.

En la figura 4 se observa el efecto de las micorrizas en la estructura de las raíces del fresno y cómo fue determinante la influencia del sustrato en el que se desarrollaron. Las primeras dos imágenes corresponden a la inoculación de Laccaria laccata en: (a) sustrato minero y (b) sustrato orgánico. Es notoria la influencia de la micorrización y el desarrollo del hongo en la raíz, principalmente por abundancia de pelos absorbentes. En el recuadro (c) se muestra el crecimiento de la raíz de las plantas inoculadas con Lactarius deliciosus en sustrato minero, pudiendo observarse la inhibición de crecimiento de micelio externo, mientras que en (d) se muestra la longitud de éste, evidenciando el efecto de la ectomicorriza. En el recuadro (e) es notable la repercusión de la falta de inoculación en el desarrollo de ciertas estructuras benéficas, principalmente la densidad de las raíces, que son las permiten la captación de agua y nutrientes. En la figura 5 se puede apreciar la densidad de raíces en una planta no inoculada y en una inoculada.

Finalmente, en cuanto al contenido de $\mathrm{Hg}$ en las plantas, se obtuvieron diferencias significativas entre 

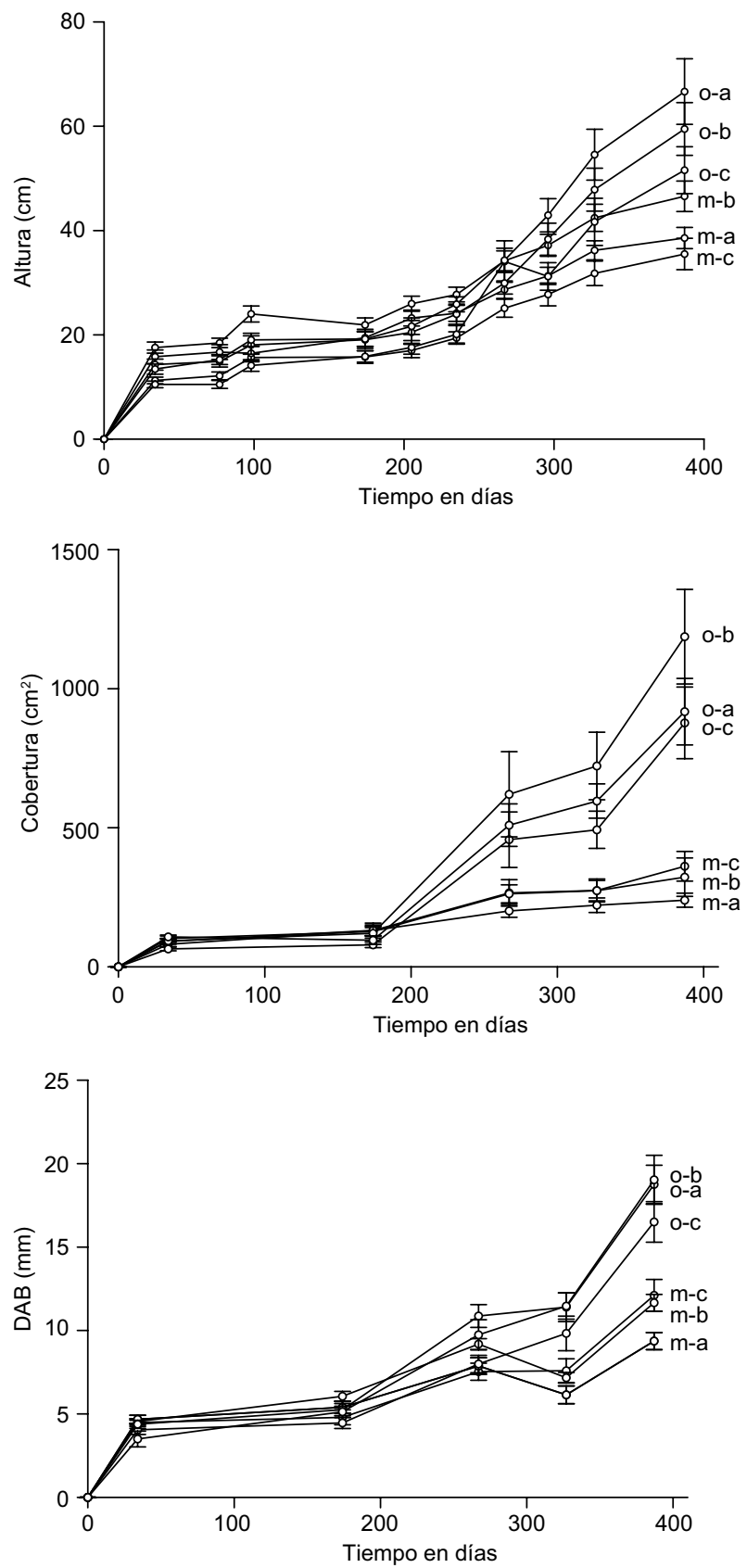

Fig. 2. Respuesta a lo largo del tiempo en altura, cobertura y diámetro a la altura de la base (DAB) de las plantas de Fraxinus uhdei inoculadas con Laccaria laccata, en los dos sustratos $(\mathrm{o}=$ orgánico; $\mathrm{m}=$ minero $)$ y las dos dosis de inoculación $\left(\mathrm{a}=1 \times 10^{6}\right.$ esporas/planta; $\mathrm{b}=2.5 \times 10^{5}$ esporas/planta)

sustratos cuando fueron inoculadas con Lactarius deliciosus $\left(\mathrm{F}_{(1,28)}=12.22 ; \mathrm{p}=0.002\right)$. El mayor contenido correspondió al sustrato minero (5.04 ppm $\pm 0.01)$ y el menor al de corteza $(5.02 \mathrm{ppm} \pm 0.01)$. Las diferencias son significativas a pesar de que las
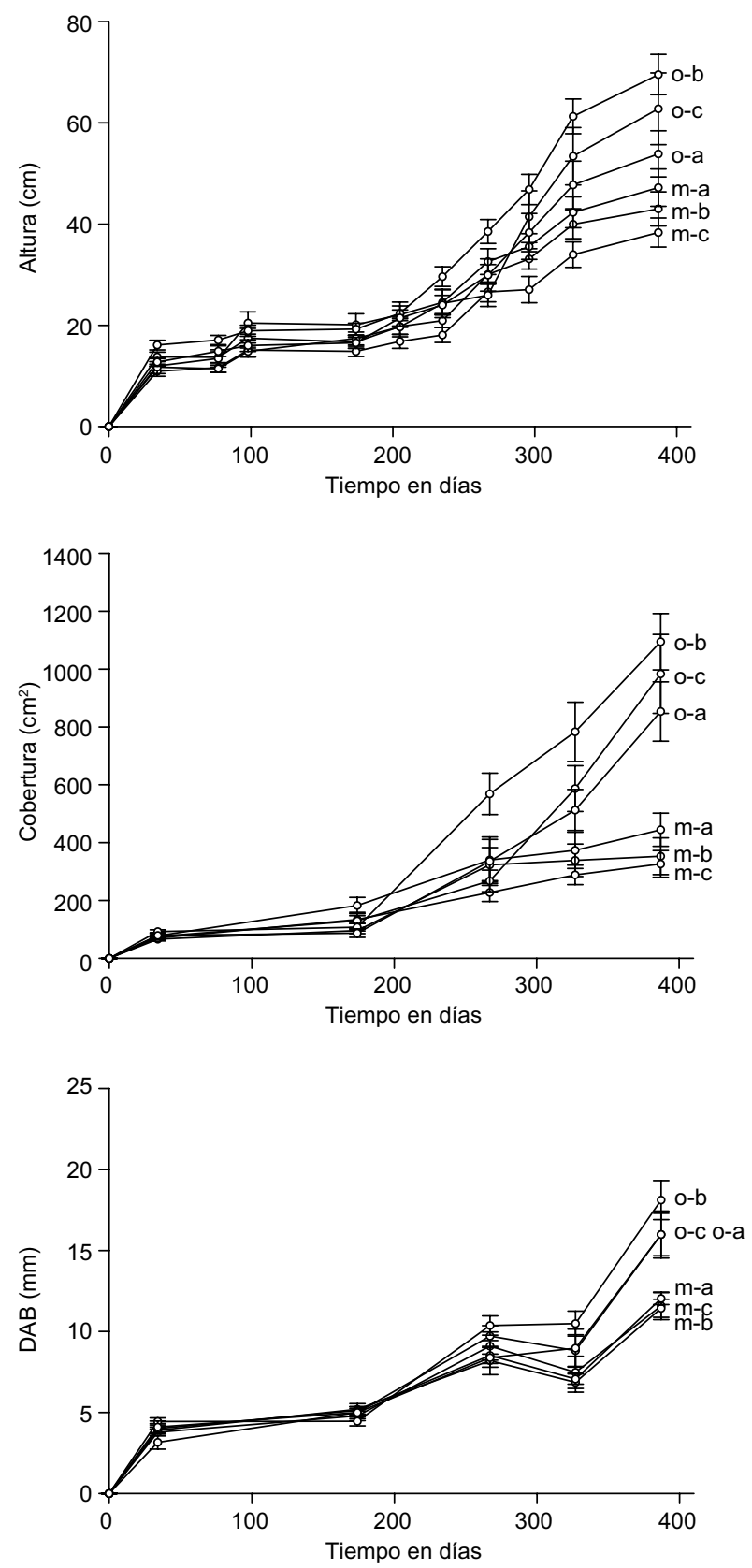

Fig. 3. Respuesta a lo largo del tiempo en altura, cobertura y diámetro a la altura de la base (DAB) de las plantas de Fraxinus uhdei inoculadas con Lactarius deliciosus, en los dos sustratos (o = orgánico; $\mathrm{m}=$ minero) y las dos dosis de inoculación $\left(\mathrm{a}=1 \times 10^{6}\right.$ esporas/planta; $\mathrm{b}=2.5$ $\times 10^{5}$ esporas/planta)

concentraciones difieren solamente en un $0.4 \%$, pero la variación para los datos de cada sustrato es muy pequeña. En plantas inoculadas con Laccaria laccata no se encontraron diferencias significativas ni para la dosis ni para el tipo de sustrato, a pesar de que el contenido es muy similar al obtenido para el otro 
CUADRO II. PROMEDIOS PARA LAS VARIABLES DE RESPUESTA CORRESPONDIENTES A LA ÚLTIMA FECHA DE MEDICIÓN DE LAS PLANTAS DE Fraxinus uhdei PARA TODOS LOS TRATAMIENTOS

\begin{tabular}{|c|c|c|c|c|c|c|}
\hline & \multicolumn{3}{|c|}{ Sustrato minero } & \multicolumn{3}{|c|}{ Sustrato orgánico } \\
\hline & Testigo & Dosis baja & Dosis alta & Testigo & Dosis baja & Dosis alta \\
\hline \multicolumn{7}{|c|}{ Laccaria laccata } \\
\hline Altura $(\mathrm{cm})$ & $33 \pm 2.5 b$ & $42 \pm 2.6 \mathrm{ab}$ & $36 \pm 1.8 b$ & $41 \pm 4.5 \mathrm{ab}$ & $47 \pm 4.1 \mathrm{a}$ & $50 \pm 5.7 a$ \\
\hline Cobertura $\left(\mathrm{cm}^{2}\right)$ & $275 \pm 41.2 b$ & $274 \pm 36.5 b$ & $221 \pm 26.2 b$ & $492 \pm 67 \mathrm{a}$ & $722 \pm 121.4 \mathrm{a}$ & $556 \pm 69.2 \mathrm{a}$ \\
\hline $\mathrm{DAB}(\mathrm{mm})$ & $7 \pm 0.7 \mathrm{bc}$ & $7 \pm 0.3 c$ & $6 \pm 0.5 c$ & $9 \pm 1 \mathrm{ab}$ & $11 \pm 0.8 \mathrm{a}$ & $10 \pm 1 \mathrm{a}$ \\
\hline Peso fresco (g) & $22 \pm 3.6 b$ & $25 \pm 2.4 b$ & $19 \pm 2.1 b$ & $75 \pm 9.5 a$ & $92 \pm 14.5 \mathrm{a}$ & $90 \pm 14.4 \mathrm{a}$ \\
\hline Peso seco $(\mathrm{g})$ & $10 \pm 1.9 b$ & $11 \pm 1.1 \mathrm{~b}$ & $8 \pm 0.9 b$ & $29 \pm 3.7 \mathrm{a}$ & $36 \pm 6.1 \mathrm{a}$ & $36 \pm 6.1 \mathrm{a}$ \\
\hline \multicolumn{7}{|c|}{ Lactarius deliciosus } \\
\hline Altura (cm) & $38 \pm 2.8 \mathrm{c}$ & $43 \pm 3.3 b c$ & $47 \pm 3.6 b c$ & $62 \pm 7 \mathrm{ab}$ & $69 \pm 4 a$ & $53 \pm 4.5 \mathrm{abc}$ \\
\hline Cobertura $\left(\mathrm{cm}^{2}\right)$ & $353 \pm 63.4 c$ & $326 \pm 46.2 c$ & $444 \pm 57.3 b c$ & $983 \pm 136.3 a$ & $1094 \pm 97.3 \mathrm{a}$ & $853 \pm 102.3 \mathrm{ab}$ \\
\hline $\mathrm{DAB}(\mathrm{mm})$ & $11 \pm 0.8 \mathrm{c}$ & $11 \pm 0.5 b c$ & $12 \pm 0.3 \mathrm{bc}$ & $15 \pm 1.3 \mathrm{ab}$ & $18 \pm 1.2 \mathrm{a}$ & $15 \pm 1.4 \mathrm{abc}$ \\
\hline Peso fresco $(\mathrm{g})$ & $20 \pm 3 b$ & $25 \pm 2.4 b$ & $27 \pm 2.7 b$ & $73 \pm 15.5 a$ & $83 \pm 9.6 a$ & $61 \pm 10.3 \mathrm{a}$ \\
\hline Peso seco (g) & $9 \pm 1.3 \mathrm{c}$ & $9 \pm 1 \mathrm{c}$ & $12 \pm 1.2 b c$ & $29 \pm 6 a$ & $35 \pm 4.5 \mathrm{a}$ & $25 \pm 4.8 \mathrm{ab}$ \\
\hline $\begin{array}{l}\text { Contenido de clorofila } \\
(\mathrm{mg} / \mathrm{g}) \text { en ambas espe- } \\
\text { cies* }\end{array}$ & $\begin{array}{c}\text { Clorofila a } \\
0.07 \pm 0.005\end{array}$ & $\begin{array}{c}\text { Clorofila b } \\
0.04 \pm 0.003\end{array}$ & $\begin{array}{c}\text { Proporción } \mathrm{a} / \mathrm{b} \\
2.13 \pm 0.2\end{array}$ & $\begin{array}{c}\text { Clorofila a } \\
0.096 \pm 0.006\end{array}$ & $\begin{array}{c}\text { Clorofila b } \\
0.045 \pm 0.003\end{array}$ & $\begin{array}{c}\text { Proporción a/b } \\
2.087 \pm 0.06\end{array}$ \\
\hline
\end{tabular}

Los promedios no identificados con las mismas letras son significativamente diferentes $(\mathrm{p} \leq 0.05)$ de acuerdo con la prueba múltiple de medias de Tukey

*Los promedios obtenidos por la clorofila son valores calculados por sustrato sin tomar en cuenta la especie de hongo

DAB: diámetro a la altura de la base

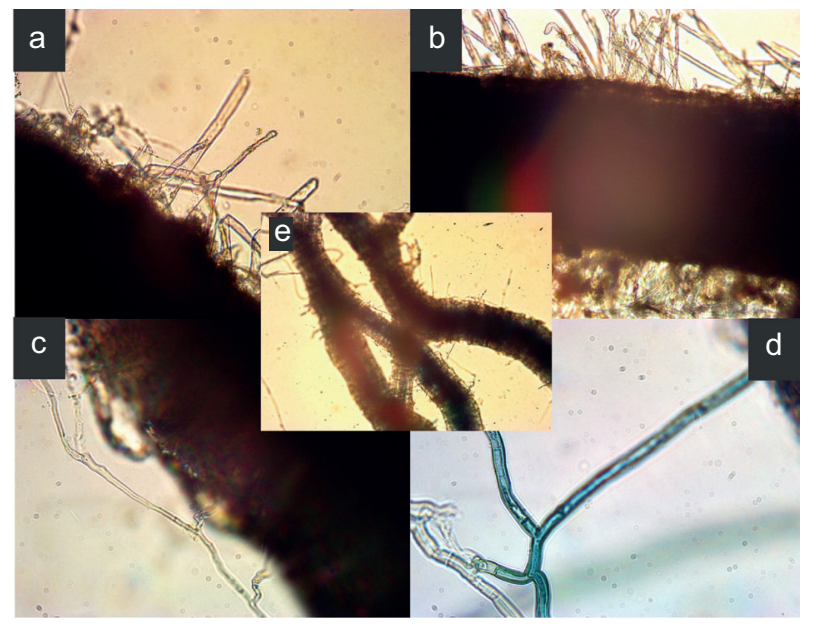

Fig. 4. (a) Raíces de Fraxinus uhdei crecidas en sustrato minero e inoculadas con la dosis alta de esporas de Laccaria laccata. (b) Raíces crecidas en sustrato orgánico inoculadas con la dosis alta de esporas de Laccaria laccata. (c) Raíces crecidas en sustrato minero inoculadas con la dosis baja de esporas de Lactarius deliciosus. (d) Micelio de Lactarius deliciosus y al centro raíces crecidas en sustrato minero sin inoculación

hongo micorrizógeno (promedio general: $5.03 \mathrm{ppm}$ ). Estos patrones merecen ser estudiados con mucho más detenimiento en experimentos adicionales.

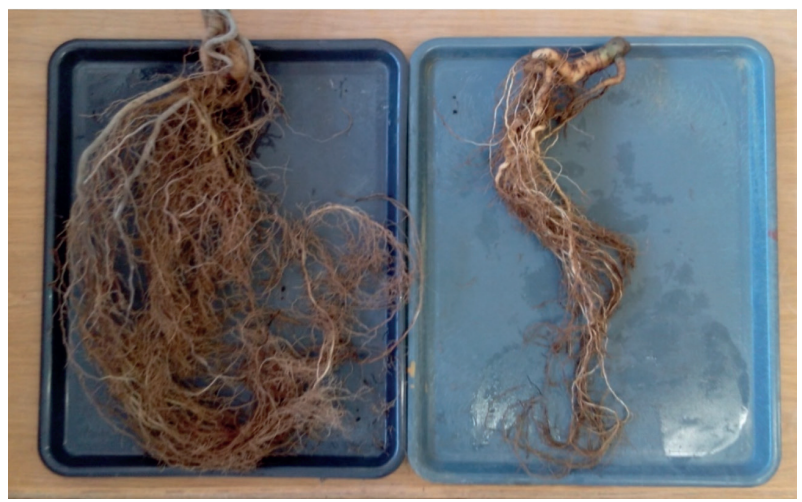

Fig. 5. Raíces de Fraxinus uhdei inoculadas (panel izquierdo) y sin inocular (panel derecho). En cuanto al contenido de clorofila-a, se presentaron diferencias estadísticamente significativas entre sustratos $\left(\mathrm{F}_{(1,165)}=5.26 ; \mathrm{p}=0.023\right)$. En el sustrato orgánico el contenido de clorofila fue de $0.096 \mathrm{mg} / \mathrm{g} \pm 0.006 \mathrm{y}$ en el sustrato minero de 0.007 $\mathrm{mg} / \mathrm{g} \pm 0.005$

\section{DISCUSIÓN}

La presente investigación mostró que la inoculación con HEM favoreció el desarrollo de las plantas de fresno tanto en el sustrato orgánico como en el minero. Este resultado concuerda con estudios 
previos sobre la inoculación de plantas con hongos micorrizógenos (Mohammadi et al. 2011). De hecho, con Pinus rigida se ha demostrado tanto este efecto como que la presencia de $\mathrm{Hg}$ no cambia significativamente la supervivencia o crecimiento, en términos de altura, diámetro y peso del brote (Crane et al. 2010).

Los resultados indicaron que las dos especies de hongos micorrizógenos incluidas en el presente estudio tienen la capacidad de establecer relaciones simbióticas con el fresno y son consistentes con estudios previos sobre la micorrización de esta especie arbórea (Báez-Pérez et al. 2015, 2017). Además, muestran que la especie arbórea y su simbionte son capaces de desarrollarse en sustratos contrastantes, uno de los cuales, el sustrato minero, se aleja considerablemente de los óptimos en términos de parámetros físicos y químicos fundamentales como la movilidad del agua, la circulación del aire, el pH, y el contenido de materia orgánica, todos fundamentales para que la relación simbiótica sea efectiva (Duddridge y Read 1984, Gast et al. 1988, Dermibas 2001, Soares et al. 2015), además de lo cual está contaminado con mercurio. Lo anterior se basa en que la presencia de estructuras propias de la micorriza en el sustrato orgánico fue más fácil de identificar y la densidad de raíces fue notoriamente diferente, aunque no se haya podido hacer una comparación cuantitativa. En el sustrato minero hubo pérdidas significativas de tejido radical por la compactación.

Además, las diferencias en la concentración de clorofila son consistentes con el hecho de que el sustrato minero es estresante para el desarrollo de las plantas, y también con el efecto positivo de la simbiosis micorrízica en el contenido de clorofila (Pedraza et al. 2001). Adicionalmente, se sabe que la disponibilidad de nutrientes, principalmente $\mathrm{N}$ y $\mathrm{Mg}$, incide sobre el contenido de clorofila, ya que estos elementos forman parte de la molécula e inciden directamente en su síntesis (Pereira et al. 2001). En contraste, las dosis de inoculación utilizadas en este experimento no arrojaron resultados significativos para ninguna de las variables medidas, por lo que se recomienda usar las dosis bajas.

Ciertas ectomicorrizas acumulan metales pesados en el micelio, por ser la estructura que tiene mayor contacto con la superficie del suelo, así como en la red de Hartig, que es el intermediario entre la solución del suelo y las raíces de la planta (Brown y Wilkins 1985, Colpaert y van Assche 1992, Crane et al. 2010). Por ejemplo, se ha que especies como Laccaria próxima y Lactarius hibbardae (Jones y Hutchinson 1986) tienen tolerancia al Ni, Cu y Hg; sin embargo, otras especies sí son susceptibles al Hg, como es el caso de
Lactarius rufus (Falandysz y Chwir 1997). En este estudio se encontró que entre las plantas de fresno inoculadas con Lactarius deliciosus y las no inoculadas hubo diferencias significativas en el contenido de $\mathrm{Hg}$ en los tejidos. Este estudio aporta información relevante para explorar el papel de las ectomicorrizas en la extracción de metales pesados, pues los resultados indican que plantas de fresno inoculadas tienen un buen desempeño en un sustrato contaminado, y soportan otras observaciones de que esta especia arbórea puede desarrollarse en suelos con escasos nutrientes (Francis 1990, Báez-Pérez et al. 2015).

Por ello, para llevar a cabo una restauración en sitios contaminados con metales pesados, es recomendable aprovechar las simbiosis, en este caso la relación del fresno con hongos ectomicorrízicos. Asimismo, es importante destacar que el primero es una especie nativa del país, lo cual aumenta las posibilidades de éxito en la repoblación del sitio, además de tenr un potencial maderable.

\section{CONCLUSIONES}

El fresno forma una simbiosis ectomicorrízica con Laccaria laccata y Lactarius deliciosus cuando es cultivado en sustratos con alto contenido de $\mathrm{Hg}$, y tiene mejor desempeño que cuando no está micorrizado. Es importante mencionar que las especies de hongos micorrizógenos usadas en este estudio son comunes en los bosques templados de México, al igual que la especie arbórea, lo que es una indicación del potencial para su utilización en proyectos de recuperación de suelos contaminados y de depósitos de residuos mineros en México.

\section{AGRADECIMIENTOS}

El presente proyecto de investigación fue financiado por la Dirección General de Asuntos del Personal Académico de la Universidad Nacional Autónoma de México a través del proyecto IN-116218 otorgado a Roberto Lindig Cisneros. Deseamos agradecer el apoyo del Dr. Luis Escalera por los análisis de mercurio en tejidos vegetales.

\section{REFERENCIAS}

Báez-Pérez A.L., Gómez-Romero M., Villegas J., de la Barrera E., Carreto-Montoya L. y Lindig-Cisneros R. (2015). Inoculación con hongos micorrícicos y 
fertilización con urea de plantas de Fraxinus uhdei en acrisoles provenientes de sitios degradados. Bot. Sci. 93 (3), 501-508. DOI: 10.17129/botsci.207

Báez-Pérez A.L., Lindig-Cisneros R. y Villegas J. (2017). Survival and growth of nursery inoculated Fraxinus uhdei in acrisol gullies. Madera Bosques 23, 7-14.

Brown M.T. y Wilkins D.A. (1985). Zinc tolerance of mycorrhizal Betula. New Phytol. 99 (1) 101-106. DOI: 10.1111/j.1469-8137.1985.tb03640.x

Brown S., de Volder P., Comptom H. y Henryl C. (2007) Effect of amendment $\mathrm{C}: \mathrm{N}$ ratio on plant richness, cover and metal content for acidic $\mathrm{Pb}$ and $\mathrm{Zn}$ mine tailings in Leadville, Colorado. Environ. Pollut. 149, 165-172. DOI: 10.1016/J.ENVPOL.2007.01.008

Colpaert J.V. y van Assche J. (1992). Zinc toxicity in ectomycorrhizal Pinus silvestris. Plant Soil 143 (2), 201-211. DOI: 10.1007/BF00007874

Conabio (1907). Especies útiles para la restauración ecológica: Fraxinus uhdei. Comisión Nacional para el Conocimiento y Uso de la Biodiversidad [en línea]. http://www.conabio.gob.mx/conocimiento/info_especies/arboles/doctos/53-oleac1m.pdf 07/01/2017.

Corona P., Uribe J.A., Razo N., Martínez M., Maldonado R., Ramos Y. y Robles J. (2010). The impact of mining in the regional ecosystem: The mining district of El Oro and Tlalpujahua, Mexico. De Re Metallica 15, 21-34.

Corona P., Maldonado R., Ramos Y.R., Robles J., Lozano R. y Martínez M. (2017). Geoquímica y mineralogía de los jales del distrito minero Tlalpujahua-El Oro, México, y sus implicaciones de impacto ambiental. Rev. Mex. Cienc. Geol. 34 (3), 250-273. DOI: 10.22201/cgeo.20072902e.2017.3.540

Crane S., Dighton J. y Barkay T. (2010). Growth responses to and accumulation of mercury by ectomycorrhizal fungi. Fungal Biol. 114 (10), 873-880.

DOI: 10.1016/j.funbio.2010.08.004

Crane S., Dighton J. y Barkay T. (2012). The effect of mercury on the establishment of Pinus rigida seedling and the development of their ectomycorrhizal communities. Fun. Ecol. 5 (2), 245-251.

DOI: 10.1016/j.funeco.2011.12.001

Crawley M.J. (2007). The R book. John Wiley and Sons, Londres, Reino Unido, 942 pp.

Dermibas A. (2001). Heavy metal bioaccumulation by mushrooms from artificially fortified soils. Food Chem. 74 (3), 293-30.

DOI: $10.1016 / \mathrm{S} 0308-8146(01) 00155-8$

Duddridge J.A. y Read D.J. (1984). The development and ultrastructure of ectomycorrhizas. II. Ectomycorrhizal development on pine in vitro. New Phytol. 96 (4), 545-553.

Eurachem Working Group. (1998) A laboratory guide to method validation and related topics. In The fitness for purpose of analytical method, Edition 1.0-1998. LGC, Teddington, ISBN 0-948926-12-0

Falandysz J. y Chwir A. (1997). The concentrations and bioconcentration factors of mercury in mushrooms from the Mierzeja Wislana sand-bar, Northern Poland. Sci. Total Environ. 203 (3), 221-228. DOI: $10.1016 / \mathrm{S} 0048-9697(97) 00150-2$

Francis J.K. (1990). Fraxinus uhdei (Wenzing) Lingelsh. Fresno tropical ash. Oleaceae olive family USDA. Forest Service. Southern Forest Experiment Station Institute of Tropical Forestry, San Juan, Puerto Rico, 4 pp.

Gast C.H., Jansen E., Bierling J. y Haantra L. (1988). Heavy metal in mushrooms and their relationship with soil characteristics. Chemosphere 17 (4), 789-799. DOI: $10.1016 / 0045-6535(88) 90258-5$

Góngora J.P. (2013). Evolución reciente de la minería en México. Comercio Exterior 63 (4), 1-5.

González W.A. (2012). Evaluación del crecimiento y desarrollo larvario de Strombus gigas en mesocosmos, una alternativa para su cultivo. Tesis de Maestría. Centro de Investigación y Estudios Avanzados, Instituto Politécnico Nacional. Mérida, Yucatán, México, 73 pp.

Jim C.Y. (2001) Ecological and landscape rehabilitation of a quarry site in Hong Kong. Rest. Ecol. 9 (1), 85-94. DOI: 10.1046/j.1526-100x.2001.009001085.x

Jones M.D. y Hutchinson T.C. (1986). The effect of mycorrhizal infection on the response of Betula papyrifera to nickel and cooper. New Phytol. 102 (3), 429-442. DOI: $10.1111 /$ j.1469-8137.1986.tb00820.x

Lacerda L.D. (1997). Global mercury emissions from gold and silver mining. Water Air Soil Poll. 97 (3-4), 209-221. DOI: $10.1023 / \mathrm{A}: 1018372505344$

Lichtenthaler H.K. (1987). Chlorophylls and carotenoids: Pigments of photosynthetic biomembranes. En: Lipids and lipid polymers in higher plants (Tevini M. y Lichtenthaler H.K., Eds.). Springer, Nueva York, EUA, 217-289. DOI: 10.1007/978-3-642-66632-2

Mohammadi K., Khalesro S., Sohrabi Y. y Heidari G. (2011). A review: Beneficial effects of the mycorrhizal fungi for plant growth. J. Appl. Environ. Biol. Sci. 1 (9), 310 -319.

Muñoz J. (1986). La minería en México. Bosquejo histórico. Quinto Centenario 11, 145-155.

Pedraza M., Jaen D., Gutiérrez A., Colinas T. y López C. (2001). Crecimiento y nutrición de micro plantas de gerbera inoculados con hongos micorrízicos arbusculares. Agrociencia 35 (2), 149-158.

Pereira G., Sánchez M., Ríos D. y Herrera M.A. (2001). Micorrizas vesículo arbusculares y su incidencia en el crecimiento de plántulas de Eucalyptus camaldulensis Dehnh. Bosque 22 (2), 39-44.

DOI: $10.4206 /$ bosque.2001.v22n2-04

Pereira G., Campos J.L., Chávez D., Anabalon L. y Arriagada C. (2014). Caracterización del crecimiento 
miceliar del hongo ectomicorrízico Lactarius aff. deliciosus y su simbiosis con plantulas de Pinus radiata. Revista de Ciencias Forestales 22 (1-2), 30-39.

Popma J., Bongers J.L. y Meave J. (1988). Patterns in the vertical structure of the tropical lowland rainforest of Los Tuxtlas. Vegetation 74 (1), 81-91.

DOI: $10.1007 / \mathrm{BF} 00045615$

Royo P., Fernández M. y Fischer C. R. (1998). Síntesis micorricíca de Lactarius deliciosus Fr. y Pinus silvestris L. Invest. Ag. Sist. Recur. For. 74 (1-2), 85-93.

Singh A.N., Raghubanshi A.S. y Sigh J.S. (2004). Comparative performance and restoration potential of two Albizia species planted on mine spoil in a dry tropical region, India. Ecol. Eng. 22,123-140.

DOI: 10.1016/j.ecoleng.2004.04.001

Soares L., Henrique P., Fonseca D.C., Christófano A., José M., de Barros E., Duarte V.H. y Fernandes A.L. (2015). In vitro evaluation of Eucalyptus ectomycorrhizae on substrate with phosphorus doses for fungal preselection. Ärvore 39 (1), 127-136.

DOI: $10.1590 / 0100-67622015000100012$
Tucker C. y Ungar I.A. (2002). Aboveground vegetation, seed bank and soil analysis of a 31-year-old forest restoration on coal mine spoil in southeastern Ohio. Am. Midl. Nat. 147, 44-59.

DOI: 10.1674/0003-0031(2002)147[0044:AVSBAS ]2.0.CO;2

Uribe J.A. (2002). La minería en Michoacán: quinientos años de su historia. En: Historia de la minería en Michoacán (Uribe J.A., Ed.). Universidad Michoacana de San Nicolás de Hidalgo. Morelia, Michoacán, México, 84-142.

Yogesh P., Mahmoud S., Lallo J. y Lang H. (2015). Sample preparation method for mercury analysis in reagent chemicals by ICP-OES. Espectroscopy 30, 8-17. 